\title{
A new Alon-Babai-Suzuki-type inequality on set systems
}

Rudy XJ Liu' and Kyung-Won Hwang ${ }^{2 *}$

"Correspondence:
khwang@dau.ac.kr
${ }^{2}$ Department of Mathematics,
Dong-A University, Busan, 604-714,
Republic of Korea
Full list of author information is
available at the end of the article

available at the end of the article

\begin{abstract}
Let $K=\left\{k_{1}, k_{2}, \ldots, k_{r}\right\}$ and $\mathcal{L}=\left\{l_{1}, I_{2}, \ldots, I_{s}\right\}$ be two sets of nonnegative integers. Let $\mathcal{F}$ be a family of subsets of $[n]$ such that $|F| \in K$ for every $F \in \mathcal{F}$ and $|E \cap F| \in \mathcal{L}$ for every pair of distinct subsets $E, F \in \mathcal{F}$. We prove that

$$
|\mathcal{F}| \leq\left(\begin{array}{c}
n-1 \\
s
\end{array}\right)+\left(\begin{array}{c}
n-1 \\
s-1
\end{array}\right)+\cdots+\left(\begin{array}{c}
n-1 \\
s-2 r+1
\end{array}\right)
$$

when we have the conditions that $K \cap \mathcal{L}=\emptyset$ and $n \geq s+\max k_{i}$. This result gives an improvement for Alon, Babai, and Suzuki's conjecture under the nonmodular. This paper gets an improvement of a theorem of Hwang et al. with $K \cap \mathcal{L}=\emptyset$.

MSC: 05D05

Keywords: Alon-Babai-Suzuki inequalities; Frankl-Ray-Chaudhuri-Wilson theorems; multilinear polynomials
\end{abstract}

\section{Introduction}

In this paper, let $X$ denote the set $[n]=\{1,2, \ldots, n\}$ and $\mathcal{L}=\left\{l_{1}, l_{2}, \ldots, l_{s}\right\}$ be a set of $s$ nonnegative integers. A family $\mathcal{F}$ of subsets of $X$ is called $\mathcal{L}$-intersecting if $|E \cap F| \in \mathcal{L}$ for every pair of distinct subsets $E, F \in \mathcal{F}$. A family $\mathcal{F}$ is $k$-uniform if it is a collection of $k$-subsets of $X$.

In 1975, Ray-Chaudhuri and Wilson proved the following fundamental result.

Theorem 1.1 (Ray-Chaudhuri and Wilson [1]) If $\mathcal{F}$ is a $k$-uniform $\mathcal{L}$-intersecting family of subsets of $X$, then

$$
|\mathcal{F}| \leq\left(\begin{array}{l}
n \\
s
\end{array}\right)
$$

In terms of the parameters $n$ and $s$, this inequality is best possible, as shown by the set of all $s$-subsets of $X$ with $\mathcal{L}=\{0,1, \ldots, s-1\}$. A nonuniform version of Theorem 1.1 was proved by Frankl and Wilson in 1981.

Theorem 1.2 (Frankl and Wilson [2]) If $\mathcal{F}$ is an $\mathcal{L}$-intersecting family of subsets of $X$, then

$$
|\mathcal{F}| \leq\left(\begin{array}{l}
n \\
s
\end{array}\right)+\left(\begin{array}{c}
n \\
s-1
\end{array}\right)+\cdots+\left(\begin{array}{l}
n \\
0
\end{array}\right)
$$

๑ 2013 Liu and Hwang; licensee Springer. This is an Open Access article distributed under the terms of the Creative Commons Attribution License (http://creativecommons.org/licenses/by/2.0), which permits unrestricted use, distribution, and reproduction in any medium, provided the original work is properly cited. 
This result is again best possible in terms of the parameters $n$ and $s$, as shown by the family of all subsets of size at most $s$ of $X$ with $\mathcal{L}=\{0,1, \ldots, s-1\}$.

In 1991, Alon, Babai, and Suzuki proved the following inequality, which is a generalization of the well-known Frankl-Ray-Chaudhuri-Wilson theorems (Theorem 1.1 and Theorem 1.2).

Theorem 1.3 (Alon, Babai, and Suzuki [3]) Let $K=\left\{k_{1}, k_{2}, \ldots, k_{r}\right\}, \mathcal{L}=\left\{l_{1}, l_{2}, \ldots, l_{s}\right\}$ be two sets of nonnegative integers and let $\mathcal{F}$ be an $\mathcal{L}$-intersecting family of subsets of $X$ such that $|F| \in K$ for every $F \in \mathcal{F}$. If $\min k_{i}>s-r$, then

$$
|\mathcal{F}| \leq\left(\begin{array}{c}
n \\
s
\end{array}\right)+\left(\begin{array}{c}
n \\
s-1
\end{array}\right)+\cdots+\left(\begin{array}{c}
n \\
s-r+1
\end{array}\right) .
$$

Note that it is best possible in terms of the parameters $n, r$, and $s$, as shown by the set of all subsets of $X$ with size at least $s-r+1$ and at most $s$, and $\mathcal{L}=\{0,1, \ldots, s-1\}$.

Since then, many Alon-Babai-Suzuki-type inequalities have been proved. Below is a list of related results in this field obtained by others.

Theorem 1.4 Let $K=\left\{k_{1}, k_{2}, \ldots, k_{r}\right\}, \mathcal{L}=\left\{l_{1}, l_{2}, \ldots, l_{s}\right\}$ be two sets of nonnegative integers and let $\mathcal{F}$ be an $\mathcal{L}$-intersecting family of subsets of $X$ such that $|F| \in K$ for every $F \in \mathcal{F}$. If $K \cap \mathcal{L}=\emptyset$, then

$$
|\mathcal{F}| \leq\left(\begin{array}{c}
n-1 \\
s
\end{array}\right)+\left(\begin{array}{c}
n-1 \\
s-1
\end{array}\right)+\cdots+\left(\begin{array}{c}
n-1 \\
0
\end{array}\right) .
$$

Theorem 1.5 (Snevily [4]) Let $K=\left\{k_{1}, k_{2}, \ldots, k_{r}\right\}, \mathcal{L}=\left\{l_{1}, l_{2}, \ldots, l_{s}\right\}$ be two sets of nonnegative integers and let $\mathcal{F}$ be an $\mathcal{L}$-intersecting family of subsets of $X$ such that $|F| \in K$ for every $F \in \mathcal{F}$. If $\min k_{i}>\max l_{j}$, then

$$
|\mathcal{F}| \leq\left(\begin{array}{c}
n-1 \\
s
\end{array}\right)+\left(\begin{array}{c}
n-1 \\
s-1
\end{array}\right)+\cdots+\left(\begin{array}{c}
n-1 \\
s-2 r+1
\end{array}\right) .
$$

Theorem 1.6 (Hwang and Sheikh [5]) Let $K=\{k, k+1, \ldots, k+r-1\}, \mathcal{L}=\left\{l_{1}, l_{2}, \ldots, l_{s}\right\}$ be two sets of nonnegative integers and let $\mathcal{F}$ be an $\mathcal{L}$-intersecting family of subsets of $X$ such that $|F| \in K$ for every $F \in \mathcal{F}$. If $K \cap \mathcal{L}=\emptyset$ and $k>s-r$, then

$$
|\mathcal{F}| \leq\left(\begin{array}{c}
n-1 \\
s
\end{array}\right)+\left(\begin{array}{c}
n-1 \\
s-1
\end{array}\right)+\cdots+\left(\begin{array}{c}
n-1 \\
s-r
\end{array}\right)
$$

Theorem 1.7 (Hwang and Sheikh [5]) Let $K=\left\{k_{1}, k_{2}, \ldots, k_{r}\right\}, \mathcal{L}=\left\{l_{1}, l_{2}, \ldots, l_{s}\right\}$ be two sets of nonnegative integers and let $\mathcal{F}$ be an $\mathcal{L}$-intersecting family of subsets of $X$ such that $|F| \in K$ for every $F \in \mathcal{F}$. If $\bigcap_{F \in \mathcal{F}} F \neq \emptyset$ and $\min k_{i}>s-r$, then

$$
|\mathcal{F}| \leq\left(\begin{array}{c}
n-1 \\
s
\end{array}\right)+\left(\begin{array}{c}
n-1 \\
s-1
\end{array}\right)+\cdots+\left(\begin{array}{c}
n-1 \\
s-r
\end{array}\right)
$$

Conjecture 1.8 (Alon, Babai, and Suzuki [3]) Let $K$ and $\mathcal{L}$ be subsets of $\{0,1, \ldots, p-1\}$ such that $K \cap \mathcal{L}=\emptyset$, where $p$ is a prime and $\mathcal{F}=\left\{F_{1}, F_{2}, \ldots, F_{m}\right\}$ be a family of subsets of $[n]$ such that $\left|F_{i}\right|(\bmod p) \in K$ for all $F_{i} \in \mathcal{F}$ and $\left|F_{i} \cap F_{j}\right|(\bmod p) \in \mathcal{L}$ for $i \neq j$. If $n \geq s+\max k_{i}$ for every $i$, then $|\mathcal{F}| \leq\left(\begin{array}{c}n \\ s\end{array}\right)+\left(\begin{array}{c}n \\ s-1\end{array}\right)+\cdots+\left(\begin{array}{c}n \\ s-r+1\end{array}\right)$. 
In the following paper, they prove the above conjecture in the nonmodular.

Theorem 1.9 (Hwang et al. [6]) Let $K=\left\{k_{1}, k_{2}, \ldots, k_{r}\right\}, \mathcal{L}=\left\{l_{1}, l_{2}, \ldots, l_{s}\right\}$ be two sets of nonnegative integers and let $\mathcal{F}$ be an $\mathcal{L}$-intersecting family of subsets of $X$ such that $|F| \in K$ for every $F \in \mathcal{F}$. If $n \geq s+\max k_{i}$, then

$$
|\mathcal{F}| \leq\left(\begin{array}{c}
n \\
s
\end{array}\right)+\left(\begin{array}{c}
n \\
s-1
\end{array}\right)+\cdots+\left(\begin{array}{c}
n \\
s-r+1
\end{array}\right) .
$$

In this paper, we will prove the following Alon-Babai-Suzuki-type inequality which gives an improvement for Theorem 1.9 with $K \cap \mathcal{L}=\emptyset$ and for Alon, Babai and Suzuki's conjecture in the nonmodular.

Theorem 1.10 Let $K=\left\{k_{1}, k_{2}, \ldots, k_{r}\right\}, \mathcal{L}=\left\{l_{1}, l_{2}, \ldots, l_{s}\right\}$ be two sets of nonnegative integers and let $\mathcal{F}$ be an $\mathcal{L}$-intersecting family of subsets of $X$ such that $|F| \in K$ for every $F \in \mathcal{F}$. If $K \cap \mathcal{L}=\emptyset$ and $n \geq s+\max k_{i}$, then

$$
|\mathcal{F}| \leq\left(\begin{array}{c}
n-1 \\
s
\end{array}\right)+\left(\begin{array}{c}
n-1 \\
s-1
\end{array}\right)+\cdots+\left(\begin{array}{c}
n-1 \\
s-2 r+1
\end{array}\right) .
$$

We note that in some cases the conditions $K \cap \mathcal{L}=\emptyset$ and $n \geq s+\max k_{i}$ in Theorem 1.10 holds, but Snevily's condition $\min k_{i}>\max l_{j}$ in Theorem 1.5 does not. For instance, if $n=12, K=\{3,6\}$ and $\mathcal{L}=\{0,1,2,4,5\}$, then it is clear that $K \cap \mathcal{L}=\emptyset$ and $s+\max k_{i}=$ $5+6<12=n$, but $\min k_{i}=3<5=\max l_{j}$.

If the condition $K \cap \mathcal{L}=\emptyset$ in Theorem 1.10 is replaced by $\bigcap_{F \in \mathcal{F}} F \neq \emptyset$, we have the following result which gives a better bound than Theorem 1.10 and Theorem 1.7.

Theorem 1.11 Let $K=\left\{k_{1}, k_{2}, \ldots, k_{r}\right\}, \mathcal{L}=\left\{l_{1}, l_{2}, \ldots, l_{s}\right\}$ be two sets of nonnegative integers and let $\mathcal{F}$ be an $\mathcal{L}$-intersecting family of subsets of $X$ such that $|F| \in K$ for every $F \in \mathcal{F}$. If $\bigcap_{F \in \mathcal{F}} F \neq \emptyset$ and $n \geq s+\max k_{i}$, then

$$
|\mathcal{F}| \leq\left(\begin{array}{c}
n-1 \\
s
\end{array}\right)+\left(\begin{array}{c}
n-1 \\
s-1
\end{array}\right)+\cdots+\left(\begin{array}{c}
n-1 \\
s-r+1
\end{array}\right)
$$

\section{Proofs of theorems}

In this section, we will give a proof for Theorem 1.10, which is motivated by the methods used in [3-7].

Proof of Theorem 1.10 Let $\mathcal{F}=\left\{F_{1}, F_{2}, \ldots, F_{m}\right\}$. We may assume (after relabeling) that for $1 \leq i \leq t, n \in F_{i}$ and that for $i>t, n \notin F_{i}$. With each set $F_{i} \in \mathcal{F}$, we associate its characteristic vector $v_{i}=\left(v_{i_{1}}, \ldots, v_{i_{n}}\right) \in \mathbb{R}^{n}$, where $v_{i_{j}}=1$ if $j \in F_{i}$ and $v_{i_{j}}=0$ otherwise. Let $\overline{v_{i}}$ be the characteristic vector of $F_{i}^{c}$.

Recall that a polynomial in $n$ variables is multilinear if its degree in each variable is at most 1 . Let us restrict the domain of the polynomials we will work with to the $n$-cube $\Omega=\{0,1\}^{n} \subseteq \mathbb{R}^{n}$. Since in this domain $x_{i}{ }^{2}=x_{i}$ for each variable, every polynomial in our proof is multilinear.

For each $F_{i} \in \mathcal{F}$, define

$$
f_{i}(x)=\prod_{l_{j} \in \mathcal{L}}\left(v_{i} \cdot x-\left(\left|F_{i}\right|-l_{j}\right)\right) .
$$


Then $f_{i}\left(\overline{v_{i}}\right) \neq 0$ for every $1 \leq i \leq m$ and $f_{i}\left(\overline{v_{j}}\right)=0$ for $i \neq j$, since $K \cap \mathcal{L}=\emptyset$. Thus, $\left\{f_{i}(x) \mid 1 \leq\right.$ $i \leq m\}$ is a linearly independent family.

Let $\mathcal{G}=\left\{G_{1}, \ldots, G_{p}\right\}$ be the family of subsets of $X$ with size at most $s$ that contain $n$, which is ordered by size, that is, $\left|G_{i}\right| \leq\left|G_{j}\right|$ if $i<j$, where $p=\sum_{i=0}^{s-1}\left(\begin{array}{c}n-1 \\ i\end{array}\right)$. Let $u_{i}$ denote the characteristic vector of $G_{i}$. For $i=1, \ldots, p$, we define

$$
g_{i}(x)=\prod_{j \in G_{i}} x_{j}
$$

Since $g_{i}\left(u_{i}\right) \neq 0$ for every $1 \leq i \leq p$ and $g_{i}\left(u_{j}\right)=0$ for any $j<i,\left\{g_{i}(x) \mid 1 \leq i \leq p\right\}$ is a linearly independent family.

Let $\mathcal{H}=\left\{H_{1}, \ldots, H_{q}\right\}$ be the family of subsets of $[n]-\{n\}$ with size at most $s-2 r$, where $q=\sum_{i=0}^{s-2 r}\left(\begin{array}{c}n-1 \\ i\end{array}\right)$. We order the members of $\mathcal{H}$ such that $\left|H_{i}\right| \leq\left|H_{j}\right|$ if $i<j$. Let $w_{i}$ be the characteristic vector of $H_{i}$.

Let $W=\left\{n-k_{i}-1 \mid k_{i} \in K\right\} \cup\left\{n-k_{i} \mid k_{i} \in K\right\}$. Then $|W| \leq 2 r$. Set

$$
f(x)=\prod_{h \in W}\left(\sum_{j=1}^{n-1} x_{j}-h\right)
$$

For $i=1, \ldots, q$, define

$$
h_{i}(x)=f(x) \prod_{j \in H_{i}} x_{j}
$$

Note that $h_{i}\left(w_{j}\right)=0$ for any $j>i$ and $h_{i}\left(w_{i}\right) \neq 0$ for every $1 \leq i \leq q$ since $n \geq s+\max k_{i}$, and thus $\left\{h_{i}(x) \mid 1 \leq i \leq q\right\}$ is a linearly independent family.

We will show that the polynomials in

$$
\left\{f_{i}(x) \mid 1 \leq i \leq m\right\} \cup\left\{g_{i}(x) \mid 1 \leq i \leq p\right\} \cup\left\{h_{i}(x) \mid 1 \leq i \leq q\right\}
$$

are linearly independent. Suppose that we have a linear combination of these polynomials that equals zero:

$$
\sum_{i=1}^{m} \alpha_{i} f_{i}(x)+\sum_{i=1}^{p} \beta_{i} g_{i}(x)+\sum_{i=1}^{q} \gamma_{i} h_{i}(x)=0
$$

We will prove that the coefficients must be zero. First substitute the characteristic vector $\overline{v_{i}}$ of $F_{i}^{c}$ with $n \in F_{i}$ into equation (2.1). Since $n \in G_{j}, g_{j}\left(\overline{v_{i}}\right)=0$ for every $1 \leq j \leq p$. Note that we have $h_{j}\left(\overline{v_{i}}\right)=0$ for every $1 \leq j \leq q$. Recall that $f_{j}\left(\overline{v_{i}}\right)=0$ for $j \neq i$, we obtain $\alpha_{i} f_{i}\left(\overline{v_{i}}\right)=0$. Thus, $\alpha_{i}=0$ for every $1 \leq i \leq t$, since $f_{i}\left(\overline{v_{i}}\right) \neq 0$. It follows that

$$
\sum_{i=t+1}^{m} \alpha_{i} f_{i}(x)+\sum_{i=1}^{p} \beta_{i} g_{i}(x)+\sum_{i=1}^{q} \gamma_{i} h_{i}(x)=0
$$

Then we substitute the characteristic vector ${\overline{v_{i}}}^{\star}$ of $F_{i}^{c}-\{n\}$ with $n \notin F_{i}$ into equation (2.2). For every $1 \leq j \leq q$ and $1 \leq k \leq p, h_{j}\left(\overline{v_{i}}\right)=0, g_{k}\left(\overline{v_{i}}{ }^{\star}\right)=0$. Note that $f_{j}\left(\overline{v_{i}}{ }^{\star}\right)=0$ for $j \neq i$, we 
obtain $\alpha_{i} f_{i}\left({\overline{v_{i}}}^{\star}\right)=0$. Since $f_{i}\left(\overline{v_{i}}\right) \neq 0, \alpha_{i}=0$ for every $t+1 \leq i \leq m$. Therefore, equation (2.2) reduces to

$$
\sum_{i=1}^{p} \beta_{i} g_{i}(x)+\sum_{i=1}^{q} \gamma_{i} h_{i}(x)=0 .
$$

First, we substitute the characteristic vector $w_{i}$ of $H_{i}$ with the smallest size into equation (2.3). We follow the same process to substitute the characteristic vector $w_{i}$ of $H_{i}$ with the smallest size after deleting first $H_{i}$. Note that $h_{i}\left(w_{j}\right)=0$ for any $j>i$ and $h_{i}\left(w_{i}\right) \neq 0$ for every $1 \leq i \leq q$, since $H_{i}$ does not contain $n$ and $G_{i}$ contains $n, g_{k}\left(w_{i}\right)=0$ for $1 \leq k \leq p$. Thus, we reduce (2.3) to

$$
\sum_{i=1}^{p} \beta_{i} g_{i}(x)=0
$$

We prove that $\left\{g_{i}(x) \mid 1 \leq i \leq p\right\}$ is already a linearly independent family. To complete the proof, simply note that each polynomial in $\left\{f_{i}(x) \mid 1 \leq i \leq m\right\} \cup\left\{g_{i}(x) \mid 1 \leq i \leq p\right\} \cup\left\{h_{i}(x) \mid 1 \leq\right.$ $i \leq q\}$ can be written as a linear combination of the multilinear polynomials of degree at most $s$. The space of such multilinear polynomials has dimension $\sum_{i=0}^{s}\left(\begin{array}{c}n \\ i\end{array}\right)$. It follows that

$$
m+p+q=|\mathcal{F}|+\sum_{i=0}^{s-1}\left(\begin{array}{c}
n-1 \\
i
\end{array}\right)+\sum_{i=0}^{s-2 r}\left(\begin{array}{c}
n-1 \\
i
\end{array}\right) \leq \sum_{i=0}^{s}\left(\begin{array}{l}
n \\
i
\end{array}\right)
$$

which implies

$$
|\mathcal{F}| \leq\left(\begin{array}{c}
n-1 \\
s
\end{array}\right)+\left(\begin{array}{c}
n-1 \\
s-1
\end{array}\right)+\cdots+\left(\begin{array}{c}
n-1 \\
s-2 r+1
\end{array}\right) .
$$

This completes the proof of the theorem.

Proof of Theorem 1.11 Let $n \in \bigcap_{F \in \mathcal{F}} F$. Then consider $\mathcal{F}^{*}=\left\{F_{1}^{*}, F_{2}^{*}, \ldots, F_{m}^{*}\right\}$ where $F_{i}^{*}=F_{i} \backslash$ $\{n\}$ for $1 \leq i \leq m$. Now, $\left|F_{i}^{*}\right| \in K^{*}=\left\{k_{1}^{*}, k_{2}^{*}, \ldots, k_{r}^{*}\right\}$, where $k_{i}^{*}=k_{i}-1$. Similarly, $\left|F_{i}^{*} \cap F_{j}^{*}\right| \in$ $\mathcal{L}^{*}=\left\{l_{1}^{*}, l_{2}^{*}, \ldots, l_{s}^{*}\right\}$, where $l_{i}^{*}=l_{i}-1$. Since $n \geq s+\max k_{i}$, then $n-1 \geq s+\max k_{i}^{*}$. Thus, it follows from Theorem 1.9 that

$$
m \leq\left(\begin{array}{c}
n-1 \\
s
\end{array}\right)+\left(\begin{array}{c}
n-1 \\
s-1
\end{array}\right)+\cdots+\left(\begin{array}{c}
n-1 \\
s-r+1
\end{array}\right)
$$

\section{Authors' contributions}

KWH made the first polynomial and proved the first polynomials are linear independent. RXJL made the other polynomials and proved that the other polynomials are linearly independent. All authors read and approved the final manuscript.

\section{Author details}

${ }^{1}$ Department of Mathematics, Pearl River College, Tianjin University of Finance \& Economics, Tianjin, 301811, P.R. China.

2Department of Mathematics, Dong-A University, Busan, 604-714, Republic of Korea. 


\section{Acknowledgements}

This work was supported by the Dong-A University research fund.

Received: 1 February 2013 Accepted: 2 April 2013 Published: 15 April 2013

\section{References}

1. Ray-Chaudhuri, DK, Wilson, RM: On t-designs. Osaka J. Math. 12, 737-744 (1975)

2. Frankl, P, Wilson, RM: Intersection theorems with geometric consequences. Combinatorica 1, 357-368 (1981)

3. Alon, N, Babai, L, Suzuki, H: Multilinear polynomials and Frankl-Ray-Chaudhuri-Wilson-Type intersection theorems. J. Comb. Theory, Ser. A 58, 165-180 (1991)

4. Snevily, HS: A generalization of the Ray-Chaudhuri-Wilson theorem. J. Comb. Des. 3, 349-352 (1995)

5. Hwang, K-W, Sheikh, NN: Intersection families and Snevily's conjecture. Eur. J. Comb. 28, 843-847 (2007)

6. Hwang, K-W, Kim, T, Jang, LC, Kim, P, Sohn, G: Alon-Babai-Suzuki's conjecture related to binary codes in nonmodular version. J. Inequal. Appl. 2010, Article ID 546015 (2010). doi:10.1155/2010/546015

7. Snevily, HS: On generalizations of the de Bruijn-Erdös theorem. J. Comb. Theory, Ser. A 68, 232-238 (1994)

doi:10.1186/1029-242X-2013-171

Cite this article as: Liu and Hwang: A new Alon-Babai-Suzuki-type inequality on set systems. Journal of Inequalities and Applications 2013 2013:171.

\section{Submit your manuscript to a SpringerOpen ${ }^{\circ}$ journal and benefit from:}

- Convenient online submission

- Rigorous peer review

- Immediate publication on acceptance

- Open access: articles freely available online

- High visibility within the field

- Retaining the copyright to your article 